This is the peer reviewed version of the following article: Marta Rubio-Martinez ... [et al.], Freezing the nonclassical crystal growth of a coordination polymer using controlled dynamic gradients. Adv. Mater., 28: 81508155, which has been published in final form at https://doi.org/10.1002/adma.201506462

This article may be used for non-commercial purposes in accordance with

Wiley Terms and Conditions for Use of Self-Archived

Versions.

Submitted to

DOI: 10.1002/adma.

\title{
Freezing the non-classical crystal growth of a coordination polymer using controlled dynamic gradients
}

By Marta Rubio-Martinez, Inhar Imaz, Neus Domingo, Afshin Abrishamkar, Tiago Sotto Mayor, René M. Rossi, Carlos Carbonell, Andrew J. deMello, David B. Amabilino, Daniel Maspoch, ${ }^{*}$ and Josep Puigmartí-Luis ${ }^{*}$

[*] Prof. D. Maspoch

Catalan Institute of Nanoscience and Nanotechnology (ICN2), CSIC and The Barcelona Institute of Science and Technology,

Campus UAB, Bellaterra, 08193 Barcelona, Spain

Institució Catalana de Recerca i Estudis Avançats (ICREA)

08100 Barcelona, Spain.

E-mail: daniel.maspoch@icn.cat

[*] Dr. J. Puigmarti-Luis

Empa, Swiss Federal Laboratories for Materials Science and Technology

Lerchenfeldstrasse 5, CH-9014 St. Gallen, Switzerland

E-mail: josep.puigmarti@empa.ch

Dr. M. Rubio-Martinez. Dr. I. Imaz, Dr. N. Domingo, Dr. C. Carbonell

Catalan Institute of Nanoscience and Nanotechnology (ICN2), CSIC and The Barcelona Institute of Science and Technology,

Campus UAB, Bellaterra, 08193 Barcelona, Spain

A. Abrishamkar, Dr. T. Sotto Mayor, Dr. R. M. Rossi

Empa, Swiss Federal Laboratories for Materials Science and Technology

Lerchenfeldstrasse 5, CH-9014 St. Gallen, Switzerland

A. Abrishamkar, Prof. A. J. deMello

Institute of Chemical and Bioengineering

Department of Chemistry and Applied Biosciences, ETH Zurich, Zurich, Switzerland.

Prof. D. B. Amabilino

School of Chemistry, The University of Nottingham

University Park, NG7 2RD, UK.

Keywords: Coordination polymers, crystal growth, microfluidics, out-of-equilibrium structures. 
This is the peer reviewed version of the following article: Marta Rubio-Martinez ... [et al.], Freezing the nonclassical crystal growth of a coordination polymer using controlled dynamic gradients. Adv. Mater., 28: 81508155, which has been published in final form at https://doi.org/10.1002/adma.201506462

This article may be used for non-commercial purposes in accordance with

Wiley Terms and Conditions for Use of Self-Archived

Versions.

Submitted to

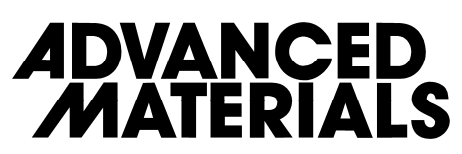

Manually engineered self-assembled structures have for many years been investigated under equilibrium conditions so that their most stable forms are reached [1-5], until recently. There has been a growing interest in obtaining and studying non-equilibrium self-assembled structures [6-9]. The primary reason for this is that non-equilibrium structures (which are typically formed transiently under a constant influx of energy)[10] can offer a broad number of intriguing opportunities in the development of novel materials and systems with advanced functionalities [11]. For example, transient and/or steady-state self-assembled structures generated far from equilibrium are the basis of many sophisticated functions observed in living systems, e.g. DNA replication and/or cell division [10]. Nonetheless, the controlled synthesis and study of intermediate, self-assembled structures is still a major challenge, which currently limits advancements in materials development and technology.

Crystals are an important type of self-assembled structures, where both long range order and control at the molecular level are central characteristics [12,13]. In contrast to living systems, where energy dissipating processes allow the appearance of adaptive and emergent functionalities, crystalline ensembles are frequently studied in their thermodynamically stable forms, where final structures are ultimately determined by chemical equilibria, diffusion and mass transport processes [14]. Even though crystals, once formed, are static structures that can be investigated at the atomic scale, it has proved difficult to establish methods that can precisely "uncover" their self-assembly process into the most thermodynamic stable forms $[15,16]$. The most frequent approach employed to understand and control the self-organization of crystalline matter involves varying the functional groups incorporated within their constituent units [17]. This heuristic approach (based on crystal engineering) has proved efficient in controlling, to some extent, the self-assembly of molecular components into intricate functional structures [18]. While there is a tremendous interest in rationalizing the crystallization process through the 
This is the peer reviewed version of the following article: Marta Rubio-Martinez ... [et al.], Freezing the nonclassical crystal growth of a coordination polymer using controlled dynamic gradients. Adv. Mater., 28: 81508155, which has been published in final form at https://doi.org/10.1002/adma.201506462

This article may be used for non-commercial purposes in accordance with

Wiley Terms and Conditions for Use of Self-Archived

Versions.

Submitted to

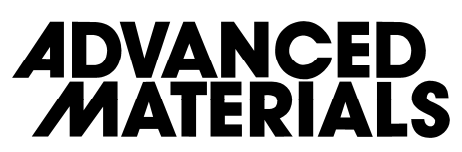

modification of the functional groups present in the molecular building blocks, there is a recognized dearth of methods and processes which allow the isolation and study of out-ofequilibrium species. In this respect, it is important to note that non-equilibrium crystal forms are not only useful in "uncovering" the self-assembly process of crystalline matter, but valuable in rationalizing new artificial materials and systems with advanced functionalities.

As crystallization is inherently a kinetic self-assembly process [15,19-21], dynamic processing technologies such as microfluidics - where molecules can react under diffusioncontrolled conditions[22-24] - can be used to control and investigate out-of-equilibrium processes. For example, controlled reaction-diffusion systems (such as those encountered in hydrodynamic flow focusing mixers)[25] can be utilized in this respect due to the precise spatial and temporal control over concentration profiles and mass transport $[26,27]$. That is, under hydrodynamic flow focusing conditions, the average residence time and the width of the reaction zone formed between two-reagent streams (where the diffusive mixing occurs) can be precisely controlled $[28,29]$. Herein, we show for the first time that reaction and/or diffusionlimited (microfluidic) environments can induce concentration gradients that facilitate the formation of novel and exceptionally ordered out-of-equilibrium structures during the crystallization of a coordination polymer (CP). In contrast to macroscopic reaction environments, we prove (both experimentally and through numerical simulations) that dynamic microfluidic conditions allow the isolation of out-of-equilibrium crystal states through the finetuning of reaction times and reagent concentration profiles. We focus our studies on the crystallization process of a $\mathrm{CP}$ because of the broad number of applications that these materials have brought onto the scene of crystalline matter [30,31].

From the vast number of CPs that could be employed in our investigations, we demonstrate the above concept by adopting a two-dimensional $\mathrm{CP}$ having the formula $\left[\mathrm{Cu}\left(4,4^{\prime}-\right.\right.$ 
This is the peer reviewed version of the following article: Marta Rubio-Martinez ... [et al.], Freezing the nonclassical crystal growth of a coordination polymer using controlled dynamic gradients. Adv. Mater., 28: 81508155, which has been published in final form at https://doi.org/10.1002/adma.201506462

This article may be used for non-commercial purposes in accordance with

Wiley Terms and Conditions for Use of Self-Archived

Versions.

Submitted to

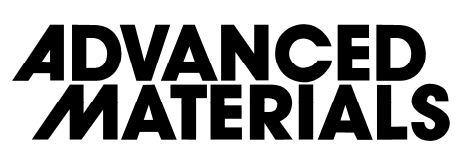

bpy)] $\left(\mathrm{NO}_{3}\right)_{2}$ (hereafter 1; where 4,4'-bpy is 4,4'-bipyridine), which is constructed by connecting $\mu_{2}$-oxo-bridged and $\mu_{2}-\mathrm{NO}_{3}$-bridged $\mathrm{Cu}$ (II) chains through 4,4'-bpy linkers (Fig. 1a and Table SI.1). 1 is particularly well-suited to the current investigation since it is easily crystallized (by diffusion of an ethanolic solution of 4,4'-bpy into an aqueous solution of $\mathrm{Cu}\left(\mathrm{NO}_{3}\right)_{2} \cdot 6 \mathrm{H}_{2} \mathrm{O}$ or by mixing the two solutions with or without stirring), and crystallizes in the form of plate-like crystals (Fig. 1b); a common crystal habit and one whose self-assembly and crystal growth development are unexplored [32].

\section{- Insert Figure 1 -}

Figure 2a shows the planar microfluidic device employed in the current investigations. This comprises four input channels and one outlet channel (see Methods Section in SI for further details). Two inlet channels are used to inject a pair of sheath flows $\left(Q_{1}\right.$ and $\left.Q_{4}\right)$ with the two other channels being used to supply the reagent solutions; one containing $\mathrm{Cu}\left(\mathrm{NO}_{3}\right)_{2} \cdot 6 \mathrm{H}_{2} \mathrm{O}\left(Q_{2}\right)$ and the other containing 4,4'-bpy linker $\left(Q_{3}\right)$. The microfluidic device was fabricated in polydimethylsiloxane (PDMS) using standard soft-lithographic methods and was covered by a glass cover slide (see Methods Section in SI for further details). We defined the four input channels and corresponding flow rates (in $\mu \mathrm{L} / \mathrm{min}$ ) as: [flow (1): $Q_{1}$, flow (2): $Q_{2}$, flow (3): $Q_{3}$, flow 4: $Q_{4}$ ]. Reactions were formed by hydrodynamically injecting a water $\left(Q_{1}\right)$, a $100 \mathrm{mM}$ aqueous solution of $\mathrm{Cu}\left(\mathrm{NO}_{3}\right)_{2} \cdot 6 \mathrm{H}_{2} \mathrm{O}\left(Q_{2}\right)$, a $100 \mathrm{mM}$ ethanolic solution of 4,4'-bpy $\left(Q_{3}\right)$, and an ethanol flow $\left(Q_{4}\right)$. The reactant concentrations were optimized to guarantee rapid crystallization whilst ensuring that microfluidic channels do not block. In initial studies, we investigated the crystallization of $\mathbf{1}$ by varying the flow-rate ratio (FRR), whilst keeping the reagent reagents flow rates $\left(Q_{2}\right.$ and $\left.Q_{3}\right)$ constant. The FRR is defined as the ratio of flow 
This is the peer reviewed version of the following article: Marta Rubio-Martinez ... [et al.], Freezing the nonclassical crystal growth of a coordination polymer using controlled dynamic gradients. Adv. Mater., 28: 81508155, which has been published in final form at https://doi.org/10.1002/adma.201506462

This article may be used for non-commercial purposes in accordance with

Wiley Terms and Conditions for Use of Self-Archived

Versions.

Submitted to

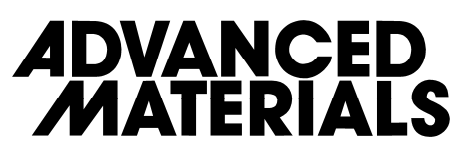

between the focusing streams and the reagent fluids (i.e. $\left.F R R=\left(\mathrm{Q}_{1}+\mathrm{Q}_{4}\right) /\left(\mathrm{Q}_{2}+\mathrm{Q}_{3}\right)\right)$. In all cases,

crystals of 1 were immediately formed at the interface between the $\mathrm{Cu}$ (II) ion and 4,4'-bpy streams after injection. The resulting crystals were collected on transmission electron microscopy grids, filter paper and/or diluted on ethanol at the end of the main channel to avoid off-chip reactions. Subsequently, the crystals formed were further characterized by FieldEmission Scanning Electron Microscopy (FE-SEM), Transmission Electron Microscopy (TEM), Polarising Optical Microscopy (POM), and X-Ray Powder Diffraction (XRPD).

\section{- Insert Figure 2-}

Figures 1c, 2c (right) and 3b (right) show typical TEM and FE-SEM images of crystals of 1 prepared at a FRR of 0.1 . These crystals that have a square plate-like habit are representative of those synthesized either by conventional diffusion or simple mixing in a macroscopic vessel (Figure 1b). This is expected since as the FRR decreases at a constant overall flow rate, the width of the diffusive mixing zone at the interface between the two reagent streams increases as a function of distance along the channel (i.e. a non-sharp concentration gradient is generated). Accordingly, the reaction zone in which the structures assemble is enlarged, thus mimics to some extent conventional diffusion on the macroscale, and assembly of the most thermodynamic stable structures will be favored. Finite element simulations strongly support the idea that a decrease in the FRR prompts an increase in the reactiondiffusion zone present along the length of the main microfluidic channel where crystallization takes place (Fig. SI.1). Crystals of $\mathbf{1}$ prepared at a FRR of 0.1 had average dimensions of 2.80 $\pm 0.52 \mu \mathrm{m}$. In addition, both the simulated (derived from the single crystal structure of $\mathbf{1}$ ) and experimental (resulting from the crystals synthesized at a FRR of 0.1) XRPD patterns are 
This is the peer reviewed version of the following article: Marta Rubio-Martinez ... [et al.], Freezing the nonclassical crystal growth of a coordination polymer using controlled dynamic gradients. Adv. Mater., 28: 81508155, which has been published in final form at https://doi.org/10.1002/adma.201506462

This article may be used for non-commercial purposes in accordance with

Wiley Terms and Conditions for Use of Self-Archived

Versions.

Submitted to

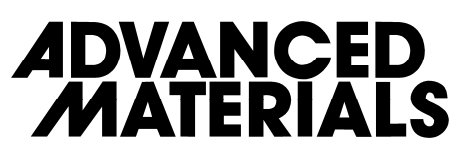

consistent (Fig. 2b and Fig. SI.2), confirming that crystals synthesized in the microfluidic

system are structurally identical to crystals prepared through conventional methods.

Additionally, it is note that $\mathbf{1}$ can be obtained as phase pure; even though the precipitation of a small amount of free 4,4'-bpy ligand is detected.

As shown in Figure 2c, we then varied the FRR from 0.1 to 5 and observed the formation of numerous and unprecedented non-equilibrium crystal morphologies. In contrast to previous studies, where blocking agents are used to study intermediate states during a crystal growth process [33], in the current investigations the ultimate shape of all generated structures solely depends upon the conditions established within the diffusive mixing zone. Increasing the FRR whilst keeping the reagent flow rates constant, a rationalized reduction of the diffusive mixing zone can be achieved (i.e. a sharp concentration gradient is generated, see Fig. SI.1), which leads to diffusion-limited and kinetically-controlled environments in which the formation of the most thermodynamic stable crystal forms can be avoided to some extent [34,35]. For example, we observed the formation of needles at a FRR of 5; needles that start to orthogonally connect through their edges at a FRR of 4; hollow frames at a FRR of 2; frames partially filled with a thinner layer at a FRR of 1; and the above-mentioned square plate-like filled crystals at a FRR of 0.1. Importantly, varying the total flow-rate (TFR) without varying the FRR provides a direct way of controlling the average residence (reaction) time for crystallization and thus throughput, but has no significant effect on the habits and structures generated (Fig. SI.3). To further understand these experimental results, numerical simulations were performed. Finite element data show that the overall concentration profiles of the reagents do not change drastically when modifying the TFR for a given FRR, but do change remarkably when varying the FRR for a given TFR. In this case, the overall concentration profiles of the reagents become narrower and the maximum concentration is reduced with increasing FRR (Fig. SI.4). This observation 
This is the peer reviewed version of the following article: Marta Rubio-Martinez ... [et al.], Freezing the nonclassical crystal growth of a coordination polymer using controlled dynamic gradients. Adv. Mater., 28: 81508155, which has been published in final form at https://doi.org/10.1002/adma.201506462

This article may be used for non-commercial purposes in accordance with

Wiley Terms and Conditions for Use of Self-Archived

Versions.

Submitted to

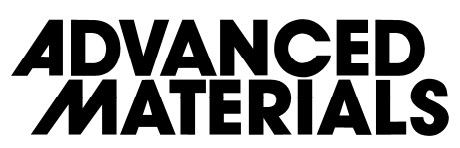

implies that the concentration of reagents present within the microfluidic channel (and which

are consumed during the formation of crystals) is reduced at higher FRRs for all TFRs considered, and thus provides for precise control of crystallization kinetics and crystal growth.

The isolated needles obtained when performing crystallization at a FRR of 5 had an average length of $500 \mathrm{~nm}$ and a diameter of $20 \mathrm{~nm}$. At a lower FRR of 4, we detected the coexistence of identical needles with some structures comprising two or three needles perpendicularly connected at their edges. Interestingly, at a FRR of 2, hollow frames, with average side dimensions of $2.95 \pm 0.71 \mu \mathrm{m}$ and edge thicknesses of $200 \mathrm{~nm}$, were found to be predominate. Further decreasing the FRR to 1 resulted in a partial filling of these hollow frames, finally forming the previously described plate-like crystals seen when a FRR of 0.1 was used.

Moreover, in contrast to other mechanistic studies where amorphous intermediate states of CPs are simply investigated by time-lapse SEM imaging analysis [36-39], in the current investigation all structures generated are crystalline. Accordingly, XRPD studies can provide valuable insights in better understanding and characterizing nanoscale self-organization of the building blocks in their isolated, non-equilibrium forms. XRPD studies were essential to confirm that all crystals generated under diffusion-limited and kinetically-controlled microfluidic environments corresponded to 1. Indeed, as shown in Figure $\mathbf{2 b}$, the XRPD patterns of all crystals obtained at different FRRs perfectly matched that simulated from the crystal structure of $\mathbf{1}$. It should be noted that XRPD patterns of the needles obtained at FRRs of 5 and 4 show broad peaks, which are attributed to their lower crystallinity.

To shed light on the growth mechanism that transforms the hollow frames $(\mathrm{FRR}=2)$ to plate-like crystals $(\mathrm{FRR}=0.1)(\mathrm{Fig} .3 \mathrm{a}, \mathrm{b})$, we further analyzed various non-equilibrium crystal forms of 1 using Atomic Force Microscopy (AFM) and POM. In the early stages of frame formation, we were able to confirm that the frames are completely hollow, with no evidence of 
This is the peer reviewed version of the following article: Marta Rubio-Martinez ... [et al.], Freezing the nonclassical crystal growth of a coordination polymer using controlled dynamic gradients. Adv. Mater., 28: 81508155, which has been published in final form at https://doi.org/10.1002/adma.201506462

This article may be used for non-commercial purposes in accordance with

Wiley Terms and Conditions for Use of Self-Archived

Versions.

Submitted to

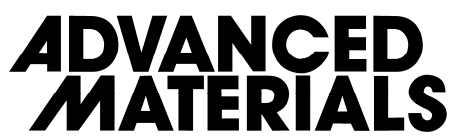

residues inside the frames (Fig. SI.5). At the vertices of the frames, the perpendicular needles

do not overlap but instead completely interpenetrate (Fig. SI.6). Additionally, POM indicates that the optical axis is the same in all sides of the frame (Fig. SI.7). The progressive filling of the internal area of the frames typically occurs by parallel needle growth, as observed in Figure 3c. For higher degrees of filling (Fig. 3d), precise observation of the filling fractions shows that needles tend to organize orthogonally in alternative growth levels. Some degree of interweaving occurs in areas where full coverage has yet to be achieved, with the needles at subsequent levels filling the gaps. Finally, at some point, needle coalescence occurs and the surface of the area inside the frames becomes uniform, ultimately forming the plate-like crystals.

\section{- Insert Figure 3 -}

The detailed mechanism leading to the formation of these ordered out-of-equilibrium structures remains unclear at the current time, however, the process seems to occur so as to lower and/or eliminate high-energy facets in the generated structures; an idea that has previously been suggested by others in regard to the shape-controlled growth of inorganic crystals [15]. Based on these previous studies with inorganic crystals and consideration of the results presented herein, we propose a dynamic crystal growth process as shown in the idealized sequence of Figure 2c. It is likely that needles isolated at a high FFR can act as seeds for the assembly of the non-equilibrium trapped intermediate states, which then evolve towards a final thermodynamic stable form: plate-like crystal structures. This proposal is supported by XRPD studies, which prove that all the structures generated have an identical chemical connectivity. Furthermore, the AFM studies support our hypothesis by confirming that growth of $\mathbf{1}$ is dynamic and that the agglomeration and progressive filling of non-equilibrium forms can occur 
This is the peer reviewed version of the following article: Marta Rubio-Martinez ... [et al.], Freezing the nonclassical crystal growth of a coordination polymer using controlled dynamic gradients. Adv. Mater., 28: 81508155, which has been published in final form at https://doi.org/10.1002/adma.201506462

This article may be used for non-commercial purposes in accordance with

Wiley Terms and Conditions for Use of Self-Archived

Versions.

Submitted to

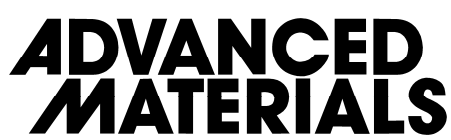

due to a parallel growth of needle-based structures. The AFM results therefore suggest that the early stage isolated seeds organize at a single level and in a perpendicular fashion, leading to the final plate-like crystalline morphologies observed in bulk and at a FFR of 0.1 (Fig. $1 \mathrm{~b}$ and 1c, respectively).

In summary, we have shown that diffusion-limited and kinetically-controlled growth regimes occurring in microfluidic devices can provide valuable insights into crystallization processes. In contrast to other methods where trapping of the structures generated during a polymerization process is achieved by taking aliquots in a controlled solvent-induced precipitation regime [40], we show, for first time, that hydrodynamic flow-focusing condition provided by the adoption of a continuous-flow microfluidic scheme can be a powerful experimental tool for the generation and isolation of non-equilibrium forms. We believe that the microfluidic-based approach presented here circumvents limitations generally ascribed to the isolation and study of transient forms during crystallization processes. We have demonstrated that microfluidic dynamic processing provides an accessible range of nonequilibrium structures present during crystal growth. These results are exciting since the control and prediction of chemical and physical properties in crystalline matter can only be achieved when methods that can precisely uncover the self-assembly process can be established. The technology presented constitutes a potential route towards a wealth of new and improved materials, where the rationalization of controlled chemical and physical properties may become reality.

\section{Experimental Section}

Materials and Methods: The reagents $\mathrm{Cu}\left(\mathrm{NO}_{3}\right)_{2} \bullet 6 \mathrm{H}_{2} \mathrm{O}$ and 4,4'-bipyridine (4,4'-bpy) were obtained from Sigma-Aldrich Co. High purity EtOH was purchased from Teknokroma. 
This is the peer reviewed version of the following article: Marta Rubio-Martinez ... [et al.], Freezing the nonclassical crystal growth of a coordination polymer using controlled dynamic gradients. Adv. Mater., 28: 81508155, which has been published in final form at https://doi.org/10.1002/adma.201506462

This article may be used for non-commercial purposes in accordance with

Wiley Terms and Conditions for Use of Self-Archived

Versions.

Submitted to

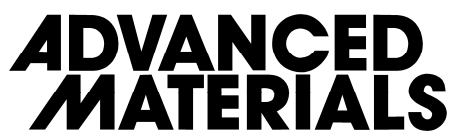

Deionised Milipore Mili-Q water was used in all experiments. Scanning electron microscopy

(SEM) images were collected on a scanning electron microscope (ZEISS EI MERLIN FE-

SEM) at acceleration voltages of $0.2-30 \mathrm{kV}$. Aluminium was used as support. Transmission electron microscopy (TEM) images were obtained with a JEOL JEM 1400 electron microscope. X-Ray EDX microanalysis was performed using an Oxford Instruments INCA energy SEM system. All measurements were performed at room temperature and at a voltage of $120 \mathrm{kV}$. Xray powder diffraction (XRPD) measurements were performed using an X'Pert PRO MPD diffractometer (Panalytical) especially configured for in-plane diffraction.

Microfluidic device fabrication: The microfluidic channels employed in this study were structured in polydimethylsiloxane (PDMS, SYLGARD® 184 Silicone Elastomer Kit) using an SU-8 (2015, Microchem) master form fabricated by standard photolithographic techniques. Before attaching the cured and structured PDMS mould to a patterned electrode surface, inlet holes connecting the microfluidic channels were punched with a Biopsy puncher. Layers were conformally contacted to form the complete microfluidic device, since this enabled removal of the PDMS layer before thermal treatment of the localized superstructures. The cross-sectional dimensions of the microchannels were $50 \mu \mathrm{m} \times 50 \mu \mathrm{m}$ for the four input microchannels, and $250 \mu \mathrm{m} \times 50 \mu \mathrm{m}$ for the main reactor channel. The total length of the main reactor channel was $9 \mathrm{~mm}$.

Synthesis of 1 via mixture of reactants: In a typical experiment, an aqueous solution of $\mathrm{Cu}\left(\mathrm{NO}_{3}\right)_{2} \cdot 6 \mathrm{H}_{2} \mathrm{O}(100 \mathrm{mM})$ was added to an ethanolic solution of 4,4'-bpy $(100 \mathrm{mM})$ with or without stirring. After a few seconds, blue crystals of 1 were formed. Anal. (\%) Calcd. for $\mathrm{C}_{20} \mathrm{H}_{18} \mathrm{Cu}_{2} \mathrm{~N}_{6} \mathrm{O}_{8}, \mathrm{NO}_{3}, \mathrm{H}_{2} \mathrm{O} ; \mathrm{C}, 35.45 ; \mathrm{H}, 2.97 ; \mathrm{N}, 14.47$. Found: C, 35.67; H, 2.69; N, 14.28. 
This is the peer reviewed version of the following article: Marta Rubio-Martinez ... [et al.], Freezing the nonclassical crystal growth of a coordination polymer using controlled dynamic gradients. Adv. Mater., 28: 81508155, which has been published in final form at https://doi.org/10.1002/adma.201506462

This article may be used for non-commercial purposes in accordance with

Wiley Terms and Conditions for Use of Self-Archived

Versions.

Submitted to

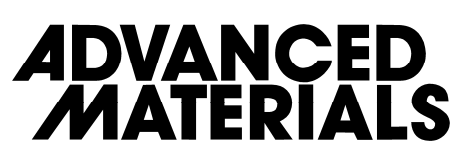

Synthesis of 1 via diffusion: In a typical experiment, a solution of 4,4'-bpy (100 mM) in ethanol and an aqueous solution of $\mathrm{Cu}\left(\mathrm{NO}_{3}\right)_{2} \bullet 6 \mathrm{H}_{2} \mathrm{O}(100 \mathrm{mM})$ were prepared. The solutions were then transferred to a test tube in a manner that generated a distinct interface between the two layers. After 4 days, dark blue crystals of 1 suitable for single-crystal XRD analysis started to form at the liquid-liquid interface. Anal. (\%) Calcd. for $\mathrm{C}_{20} \mathrm{H}_{18} \mathrm{Cu}_{2} \mathrm{~N}_{6} \mathrm{O}_{8}, \mathrm{NO}_{3}, \mathrm{H}_{2} \mathrm{O} ; \mathrm{C}, 35.45 ; \mathrm{H}, 2.97$; N, 14.47. Found: C, 35.11; H, 3.12; N, 14.28.

Synthesis of 1 using laminar flow: The syntheses of different crystal morphologies of 1 were carried out in a planar microfluidic device that consists of four input channels and one outlet channel, imprinted in PDMS and is covered by a glass plate. Reactant solutions were injected via a syringe pump system at given flows rates. We defined the flow rates (all in $\mu \mathrm{L} / \mathrm{min}$ ) using the following abbreviations: flow (1), $Q 1$; flow (2), $Q 2$; flow (3), $Q 3$; and flow (4), $Q 4$. In a typical synthetic procedure, crystals of $\mathbf{1}$ were initially prepared by injecting an aqueous solution of $\mathrm{Cu}\left(\mathrm{NO}_{3}\right)_{2} \cdot 6 \mathrm{H}_{2} \mathrm{O}(100 \mathrm{mM})$ in $Q 2$ and an ethanolic solution of 4,4'-bpy $(100 \mathrm{mM})$ in Q3. Both were accomplished by an auxiliary flow with the corresponding solvents, $Q 1$ and Q4.

X-ray crystallography: X-ray single-crystal diffraction data for $\mathbf{1}$ were collected on the BM16 Spanish line of the ESRF synchrotron in Grenoble $(\square=0.7901 \AA$ ). Data were indexed, integrated and scaled using HKL2000 software [1]. The H atoms were included in theoretical positions but not refined. The low max value is due to the data collection process, which was performed in the BM16 line with only a phi scan. The structure was solved by direct methods using the program SHELXS-97 program [2]. Refinement and all further calculations were carried out using SHELXL-97. Empirical absorption corrections were applied in both cases using SCALEPACK[1]. 
This is the peer reviewed version of the following article: Marta Rubio-Martinez ... [et al.], Freezing the nonclassical crystal growth of a coordination polymer using controlled dynamic gradients. Adv. Mater., 28: 81508155, which has been published in final form at https://doi.org/10.1002/adma.201506462

This article may be used for non-commercial purposes in accordance with

Wiley Terms and Conditions for Use of Self-Archived

Versions.

Submitted to

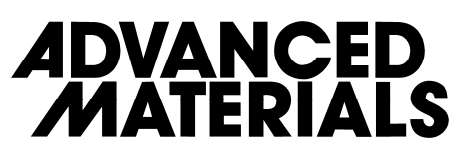

AFM measurements: Atomic Force Microscopy images were taken in amplitude modulation dynamic AFM mode, in pure non-contact conditions with an Asylum MFP3D system, using Pt coated tips (Nanosensors PPP-EFM) and a resonance frequency around $70 \mathrm{kHz}$. Images were obtained using a scanning rate of $1 \mathrm{~Hz}$ and keeping the amplitude of oscillation constant at about $50 \mathrm{~nm}$. For Kelvin Probe Force Microscopy images, an AC voltage of $1 \mathrm{~V}$ amplitude was applied to the tip at a distance of $50 \mathrm{~nm}$ to the surface, and the surface potential function difference between the tip and the sample was obtained.

Numerical simulations: The two-dimensional steady-state fluid flow and mass transport across the microfluidic device was simulated using a Finite Element approach, considering geometries and boundary-conditions as described in the manuscript. Diffusion coefficients of both reagents were assumed to be $10^{-9} \mathrm{~m}^{2} / \mathrm{s}$, in line with literature data for ethanol-water mixtures [3]. Density and dynamic viscosity of reagents and sheathed currents were assumed to be those of the corresponding pure solvents, i.e. $103 \mathrm{~kg} / \mathrm{m}^{3}$ and $8.9 \times 10^{-4} \mathrm{~Pa} \bullet$ s for water-based currents and 789 $\mathrm{kg} / \mathrm{m}^{3}$ and $1.1 \times 10^{-3} \mathrm{~Pa} \bullet$ for ethanol-based currents, respectively.

\section{Acknowledgements}

The authors acknowledge the financial support from MINECO-Spain, under projects MAT2012-30994 and CTQ2011-16009-E, and from Swiss National Science Foundation (SNF) through the project no. 200021_160174. The authors thank the Microscopy Service of the UAB. M. R. M. and C.C. thanks the ICN2 for their research fellowship, I. I., N.D. and J. P-L. thank the MINECO for RyC contracts. ICN2 acknowledges the support of the Spanish MINECO through the Severo Ochoa Centers of Excellence Program, under Grant SEV-2013-0295.

Received: ((will be filled in by the editorial staff))

Revised: ((will be filled in by the editorial staff)) Published online: ((will be filled in by the editorial staff)) 
[1] G. M. Whitesides, M. Boncheva, Proc. Natl. Acad. Sci. 2002, 99, 4769.

[2] S. S. Babu, S. Prasanthkumar, A. Ajayaghosh, Angew. Chem. Int. Ed. 2012, 51, 1766.

[3] D. Phlip, Adv. Mater. 1996, 8, 866.

[4] F. J. M. Hoeben, P. Jonkheijm, E. W. Meijer, A. P. H. J. Schenning, Chem. Rev. 2005, $105,1491$.

[5] I. C. Reynhout, J. J. L. M. Cornelissen, R. J. M. Nolte, Acc. Chem. Res. 2009, 42, 681.

[6] J. Boekhoven, J. M. Poolman, C. Maity, F. Li, L. van der Mee, C. B. Minkenberg, E. Mendes, J. H. van Esch, R. Eelkema, Nat. Chem. 2013, 5, 433.

[7] P. A. Korevaar, S. J. George, A. J. Markvoort, M. M. J. Smulders, P. A. J. Hilbers, A. P. H. J. Schenning, T. F. A. De Greef, E. W. Meijer, Nature 2012, 481, 492.

[8] B. A. Grzybowski, H. A. Stone, G. M. Whitesides, Nature 2000, 405, 1033.

[9] S. Mann, Nat. Mater. 2009, 8, 781.

[10] G. M. Whitesides, B. Grzybowski, Science 2002, 295, 2418.

[11] J. M. A. Carnall, C. A. Waudby, A. M. Belenguer, M. C. A. Stuart, J. J.-P. Peyralans, S. Otto, Science 2010, 327, 1502.

[12] J.-M. Lehn, Angew. Chem. Int. Ed. Engl. 1990, 29, 1304.

[13] O. M. Yaghi, M. O’Keeffe, N. W. Ockwig, H. K. Chae, M. Eddaoudi, J. Kim, Nature 2003, 423, 705 .

[14] J. Hulliger, Angew. Chem. Int. Ed. Engl. 1994, 33, 143.

[15] S. Mann, Angew. Chem. Int. Ed Engl. 2000, 39, 3392.

[16] D. Gebauer, M. Kellermeier, J. D. Gale, L. Bergström, H. Cölfen, Chem. Soc. Rev. $\mathbf{2 0 1 4}, 43,2348$. 
This is the peer reviewed version of the following article: Marta Rubio-Martinez ... [et al.], Freezing the nonclassical crystal growth of a coordination polymer using controlled dynamic gradients. Adv. Mater., 28: 81508155, which has been published in final form at https://doi.org/10.1002/adma.201506462

This article may be used for non-commercial purposes in accordance with

Wiley Terms and Conditions for Use of Self-Archived

Versions.

Submitted to

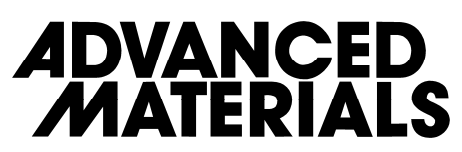

[17] M. D. Hollingsworth, Science 2002, 295, 2410.

[18] J. Kang, D. Miyajima, T. Mori, Y. Inoue, Y. Itoh, T. Aida, Science 2015, 347, 646.

[19] S. Mann, Nature 1988, 332, 119.

[20] Y. Yin, A. P. Alivisatos, Nature 2005, 437, 664.

[21] E. González, J. Arbiol, V. F. Puntes, Science 2011, 334, 1377.

[22] J. Atencia, D. J. Beebe, Nature 2005, 437, 648.

[23] R. F. Ismagilov, A. D. Stroock, P. J. A. Kenis, G. Whitesides, H. A. Stone, Appl. Phys. Lett. 2000, 76, 2376.

[24] P. J. A. Kenis, R. F. Ismagilov, G. M. Whitesides, Science 1999, 285, 83.

[25] J. B. Knight, A. Vishwanath, J. P. Brody, R. H. Austin, Phys. Rev. Lett. 1998, 80, 3863.

[26] E. Amstad, M. Gopinadhan, C. Holtze, C. O. Osuji, M. P. Brenner, F. Spaepen, D. A. Weitz, Science 2015, 349, 956.

[27] W.-Y. Lin, Y. Wang, S. Wang, H.-R. Tseng, Nano Today 2009, 4, 470.

[28] J. Puigmartí-Luis, D. Schaffhauser, B. R. Burg, P. S. Dittrich, Adv. Mater. 2010, 22, 2255.

[29] J. Puigmartí-Luis, M. Rubio-Martínez, U. Hartfelder, I. Imaz, D. Maspoch, P. S. Dittrich, J. Am. Chem. Soc. 2011, 133, 4216.

[30] S. Kitagawa, R. Kitaura, S. Noro, Angew. Chem. Int. Ed. 2004, 43, 2334.

[31] C. Janiak, Dalton Trans. 2003, 2781.

[32] T. Rodenas, I. Luz, G. Prieto, B. Seoane, H. Miro, A. Corma, F. Kapteijn, F. X. Llabrés i Xamena, J. Gascon, Nat. Mater. 2015, 14, 48.

[33] X. Sun, S. Dong, E. Wang, J. Am. Chem. Soc. 2005, 127, 13102.

[34] M. Numata, T. Kozawa, Chem. - Eur. J. 2013, 19, 12629. 
This is the peer reviewed version of the following article: Marta Rubio-Martinez ... [et al.], Freezing the nonclassical crystal growth of a coordination polymer using controlled dynamic gradients. Adv. Mater., 28: 81508155, which has been published in final form at https://doi.org/10.1002/adma.201506462

This article may be used for non-commercial purposes in accordance with

Wiley Terms and Conditions for Use of Self-Archived

Versions.

Submitted to

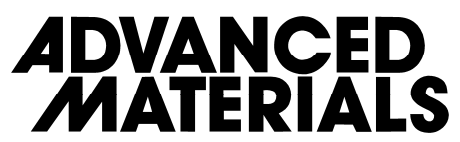

[35] M. Numata, Y. Takigami, M. Takayama, T. Kozawa, N. Hirose, Chem. - Eur. J. 2012, 18,13008

[36] M. Oh, C. A. Mirkin, Nature 2005, 438, 651.

[37] Z. Shen, G. Zhang, H. Zhou, P. Sun, B. Li, D. Ding, T. Chen, Adv. Mater. 2008, 20, 984.

[38] S. Jung, M. Oh, Angew. Chem. Int. Ed. 2008, 47, 2049.

[39] Y.-M. Jeon, G. S. Armatas, D. Kim, M. G. Kanatzidis, C. A. Mirkin, Small 2009, 5, 46.

[40] A. M. Spokoyny, D. Kim, A. Sumrein, C. A. Mirkin, Chem. Soc. Rev. 2009, 38, 1218. 
This is the peer reviewed version of the following article: Marta Rubio-Martinez ... [et al.], Freezing the nonclassical crystal growth of a coordination polymer using controlled dynamic gradients. Adv. Mater., 28: 81508155, which has been published in final form at https://doi.org/10.1002/adma.201506462

This article may be used for non-commercial purposes in accordance with

Wiley Terms and Conditions for Use of Self-Archived

Versions.

Submitted to

a)

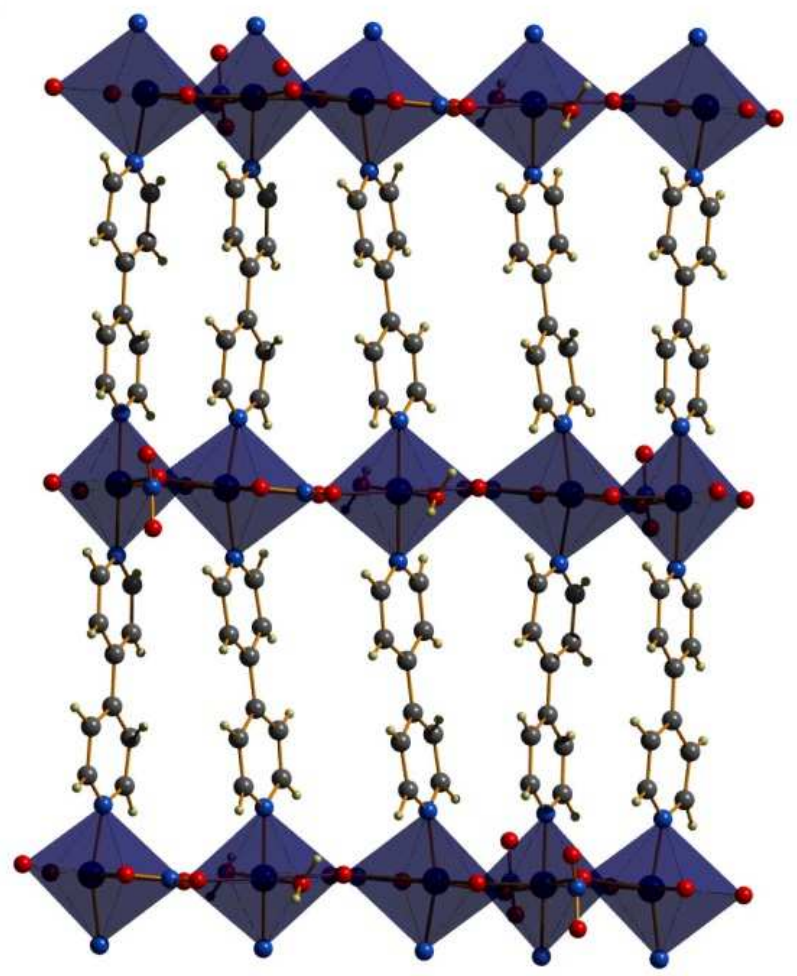

b)

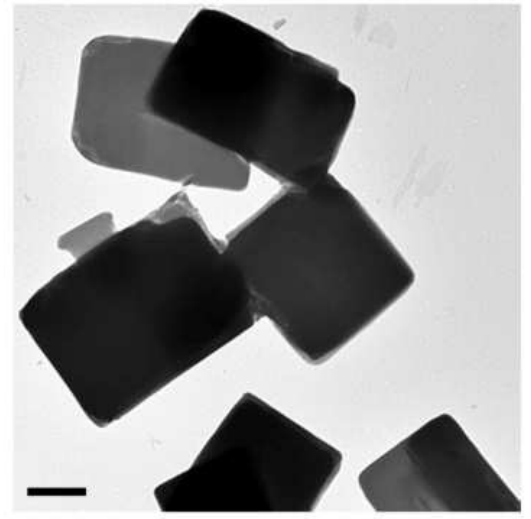

c)

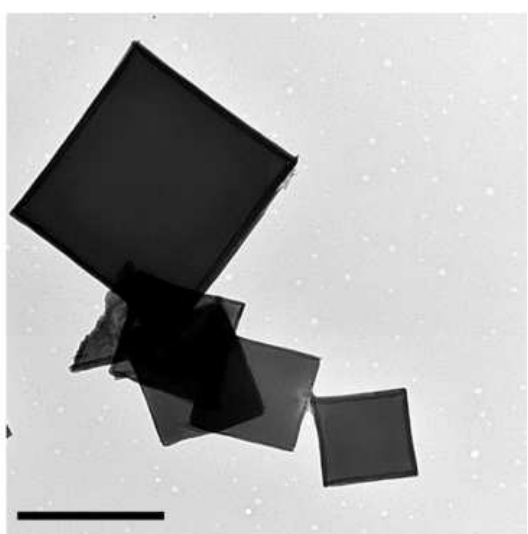

Figure 1. Coordination polymer structure. a) Crystal structure of 1. b) and c) representative TEM images showing the plate-like crystals synthesized through conventional mixing of reactants b) and under microfluidic conditions with a FRR of $0.1(\mathbf{c})$. Scale bars are $2 \mu \mathrm{m}$. 
This is the peer reviewed version of the following article: Marta Rubio-Martinez ... [et al.], Freezing the nonclassical crystal growth of a coordination polymer using controlled dynamic gradients. Adv. Mater., 28: 81508155, which has been published in final form at https://doi.org/10.1002/adma.201506462 This article may be used for non-commercial purposes in accordance with Wiley Terms and Conditions for Use of Self-Archived Versions.

Submitted to

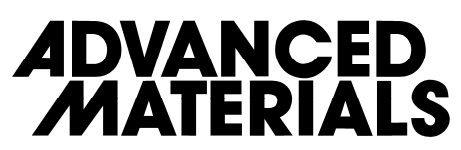

a)

b)
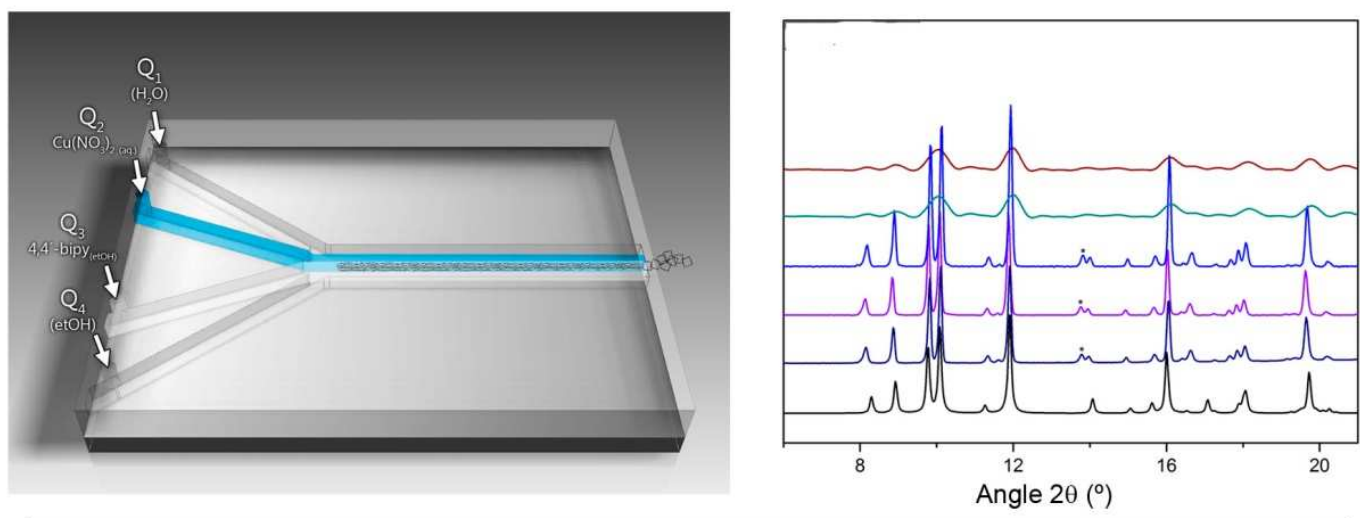

c)

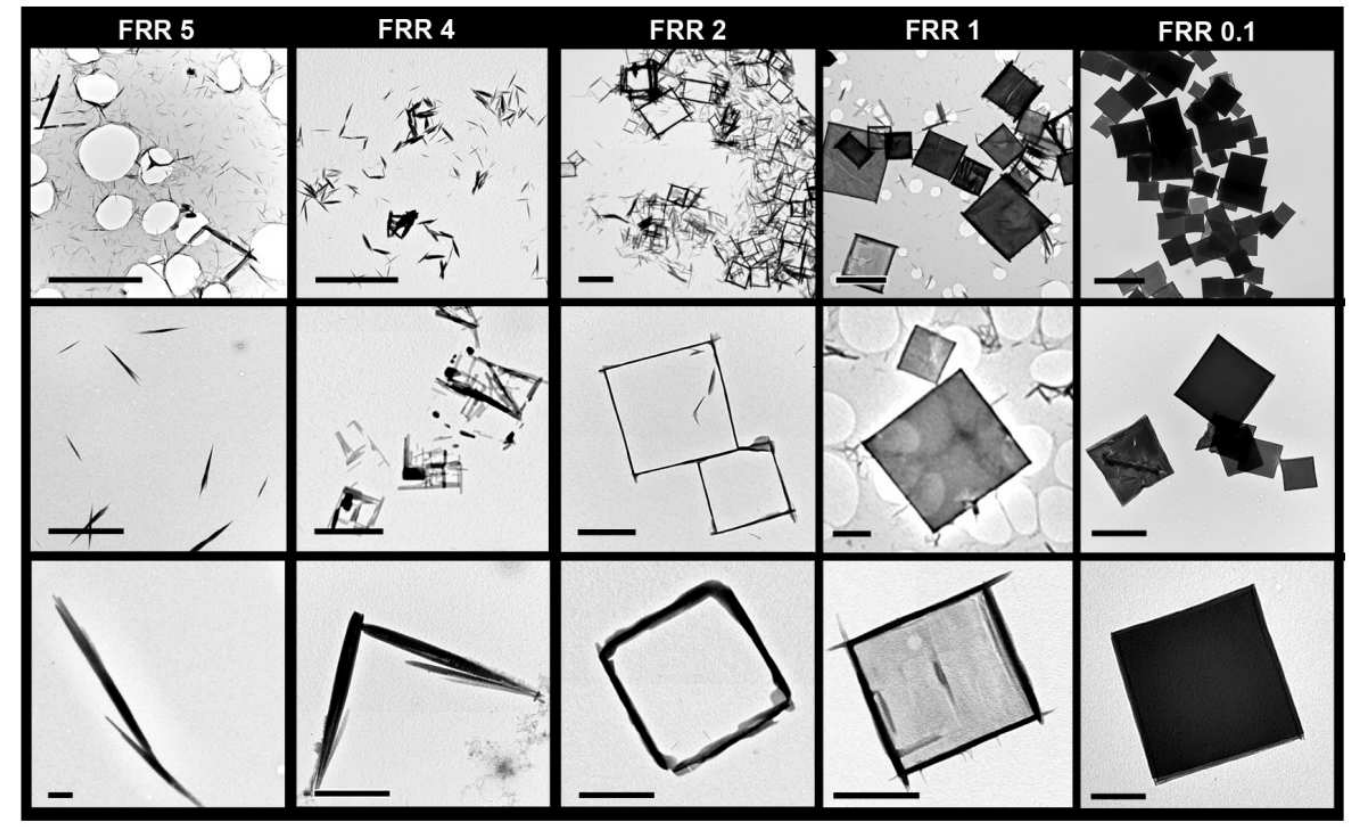

Figure 2. Morphological transitions of coordination polymer 1 using microfluidics. a)

Schematic illustration of the microfluidic device and the configuration of both reactant and sheath flows. b) XRPD patterns of 1: simulated (black), synthesized at an FRR of 0.1 (dark blue), 1 (violet), 2 (blue), 4 (green) and 5 (red). Peaks marked with asterisks correspond to the precipitation of the free 4,4'-bpy ligand. c) Sequence of TEM images of crystals of 1 fabricated in the microfluidic device at different FRRs with three different magnification levels, showing trapped crystalline phases that range from needles to hollow frames to plate-like crystals (left to right). Scale bars: $5 \mu \mathrm{m}$ (top row), $2 \mu \mathrm{m}$ (middle row) and $1 \mu \mathrm{m}$ (bottom row). 
This is the peer reviewed version of the following article: Marta Rubio-Martinez ... [et al.], Freezing the nonclassical crystal growth of a coordination polymer using controlled dynamic gradients. Adv. Mater., 28: 81508155, which has been published in final form at https://doi.org/10.1002/adma.201506462

This article may be used for non-commercial purposes in accordance with

Wiley Terms and Conditions for Use of Self-Archived

Versions.

Submitted to

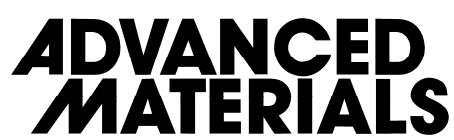

a)
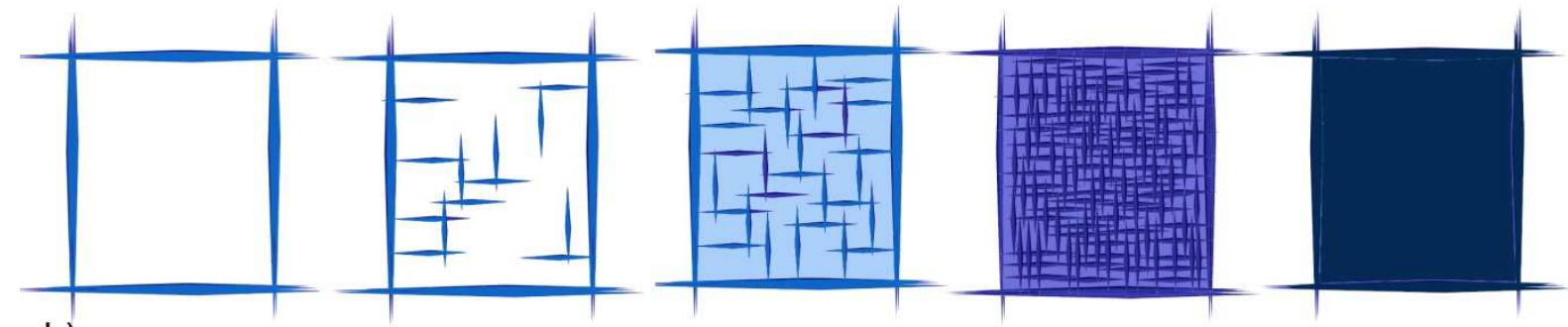

b)

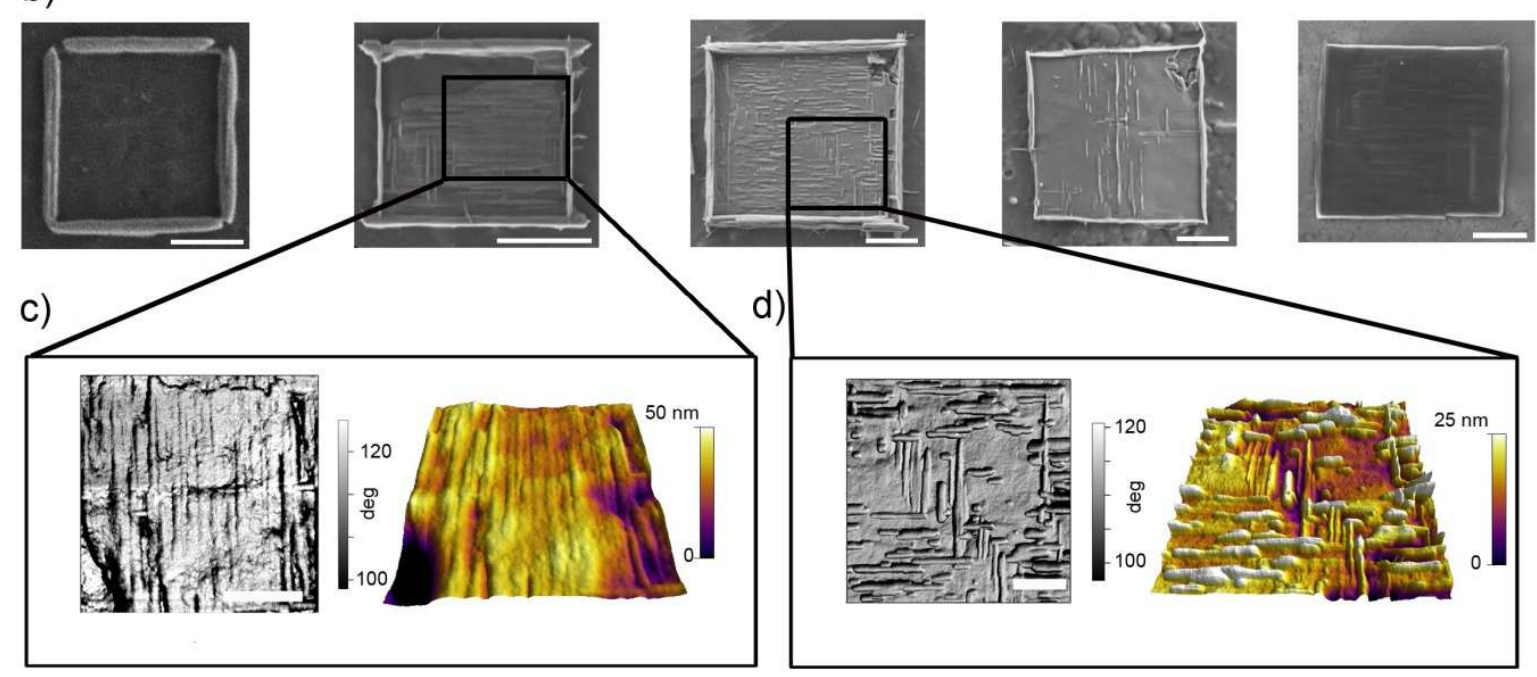

Figure 3. Evolution of crystal growth. a) Schematic representation of the crystal growth of 1 illustrating the progressive filling of the internal area of hollow frames. b) FE-SEM images showing the crystal transformation from hollow frames to plate-like crystals. c) and d) AFM images showing the crystal growth of the internal area of the frames at initial (c) and progressive (d) filling stages. The left hand side images report phase contrast, and right hand side images are 3D graphics of topography. Scale bars: $1 \mu \mathrm{m}$ 
This is the peer reviewed version of the following article: Marta Rubio-Martinez ... [et al.], Freezing the nonclassical crystal growth of a coordination polymer using controlled dynamic gradients. Adv. Mater., 28: 81508155, which has been published in final form at https://doi.org/10.1002/adma.201506462

This article may be used for non-commercial purposes in accordance with

Wiley Terms and Conditions for Use of Self-Archived

Versions.

Submitted to

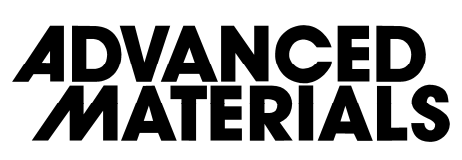

A methodology that can be efficiently used to synthesize, isolate and study out-ofequilibrium crystal structures employing controlled and diffusion-limited microfluidic environments is demonstrated. Unlike studies conducted with conventional mixing procedures in a flask, we prove experimentally and with numerical simulations that microfluidic technologies can undoubtedly fine-tune reaction times and reagents concentration profiles; factors that enable obtaining out-of-equilibrium crystal forms.

Keyword: Coordination polymers, crystal growth, microfluidics, out-of-equilibrium structures.

M. Rubio-Martínez, I. Imaz, N. Domingo, A. Abrishamkar, T. Sotto Mayor, R. M. Rossi, C.

Carbonell, A. J. deMello, D. B. Amabilino, D. Maspoch, 'J. Puigmartí-Luis*

Freezing the non-classical crystal growth of a coordination polymer using controlled dynamic gradients

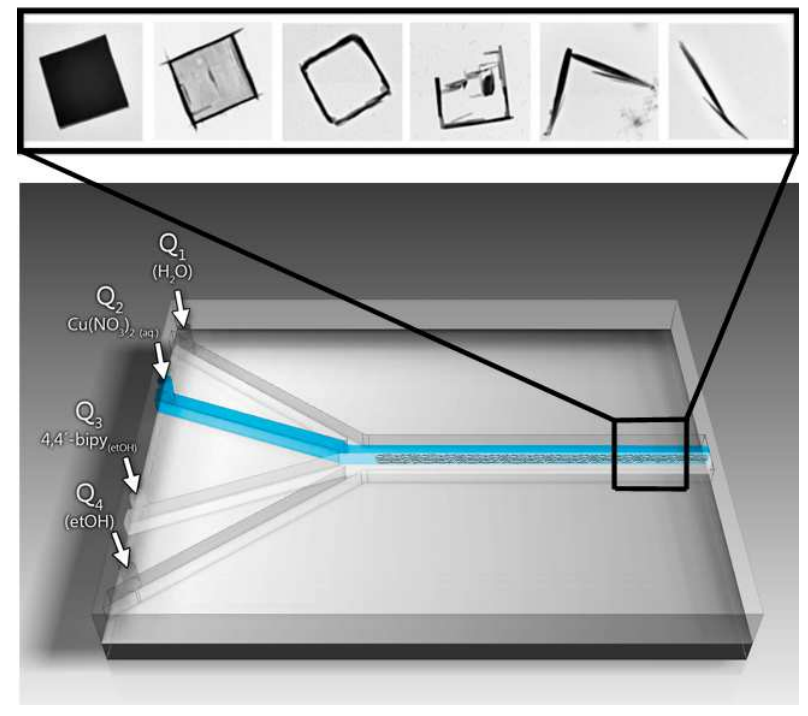




\title{
Supporting Information
}

\author{
Freezing the non-classic crystal growth of a coordination polymer by employing \\ controlled dynamic gradients
}

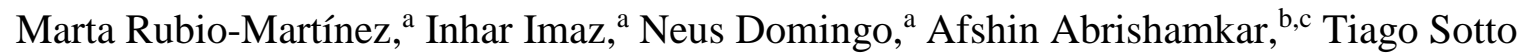
Mayor, ${ }^{\mathrm{b}}$ René Rossi, ${ }^{\mathrm{b}}$ Carlos Carbonell, ${ }^{\mathrm{a}}$ Andrew J. deMello, ${ }^{\mathrm{c}}$ David B. Amabilino, ${ }^{\mathrm{d}}$ Daniel Maspoch, ${ }^{* a, e}$ and Josep Puigmartí-Luis ${ }^{*}, \mathrm{~b}$

${ }^{a}$ Institut Catala de Nanociencia i Nanotecnologia, ICN2, Esfera UAB, Campus UAB, 08193

Bellaterra, Spain.

${ }^{b}$ Empa, Swiss Federal Laboratories for Materials Science and Technology,

Lerchenfeldstrasse 5, CH-9014 St. Gallen, Switzerland. E-mail: josep.puigmarti@empa.ch

${ }^{c}$ Institute of Chemical and Bioengineering, Department of Chemistry and Applied

Biosciences, ETH Zurich, Zurich, Switzerland

${ }^{d}$ School of Chemistry, The University of Nottingham, University Park, NG7 2RD, UK.

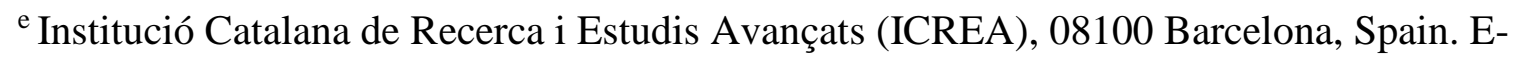
mail: daniel.maspoch@icn.cat 
This is the peer reviewed version of the following article: Marta Rubio-Martinez ... [et al.], Freezing the nonclassical crystal growth of a coordination polymer using controlled dynamic gradients. Adv. Mater., 28: 81508155, which has been published in final form at https://doi.org/10.1002/adma.201506462

This article may be used for non-commercial purposes in accordance with

Wiley Terms and Conditions for Use of Self-Archived

Versions.

Submitted to

ADNANCED

Table SI.1 | Crystal and structure refinement data.

\begin{tabular}{|c|c|}
\hline Compound & 1 \\
\hline Empirical formula & $\mathrm{C}_{20} \mathrm{H}_{18} \mathrm{Cu}_{2} \mathrm{~N}_{6} \mathrm{O}_{8}, \mathrm{NO}_{3}, \mathrm{H}_{2} \mathrm{O}$ \\
\hline Formula weight & 677.3 \\
\hline Crystal system & triclinic \\
\hline Space group & P-1 \\
\hline CCDC ref & 1059883 \\
\hline \multicolumn{2}{|l|}{ Unit cell dimensions } \\
\hline$a(\AA)$ & $10.385(4)$ \\
\hline $\mathrm{b}(\AA)$ & $11.555(4)$ \\
\hline c $(\AA)$ & $11.790(4)$ \\
\hline$\alpha(\operatorname{deg})$ & 73.91(3) \\
\hline$\beta(\operatorname{deg})$ & $84.08(3)$ \\
\hline$\gamma(\operatorname{deg})$ & $70.54(3)$ \\
\hline $\mathrm{V}\left(\AA^{3}\right)$ & $1,281.6(7)$ \\
\hline $\mathrm{Z}$ & 2 \\
\hline$F(000)$ & 686 \\
\hline Ind refln $\left(\mathrm{R}_{\text {int }}\right)$ & 4,905 (0.0199) \\
\hline$\theta_{\max }($ deg. $)$ & 27.12 \\
\hline Final $\mathrm{R}$ indices & $\mathrm{R} 1=0.0528$ \\
\hline$[\mathrm{I}>2 \sigma(\mathrm{I})]$ & $w R 2=0.1553$ \\
\hline
\end{tabular}


This is the peer reviewed version of the following article: Marta Rubio-Martinez ... [et al.], Freezing the nonclassical crystal growth of a coordination polymer using controlled dynamic gradients. Adv. Mater., 28: 81508155, which has been published in final form at https://doi.org/10.1002/adma.201506462

This article may be used for non-commercial purposes in accordance with

Wiley Terms and Conditions for Use of Self-Archived

Versions.

Submitted to

ADVANCED
MATERIALS 
This is the peer reviewed version of the following article: Marta Rubio-Martinez ... [et al.], Freezing the nonclassical crystal growth of a coordination polymer using controlled dynamic gradients. Adv. Mater., 28: 81508155, which has been published in final form at https://doi.org/10.1002/adma.201506462

This article may be used for non-commercial purposes in accordance with

Wiley Terms and Conditions for Use of Self-Archived Versions.

Submitted to
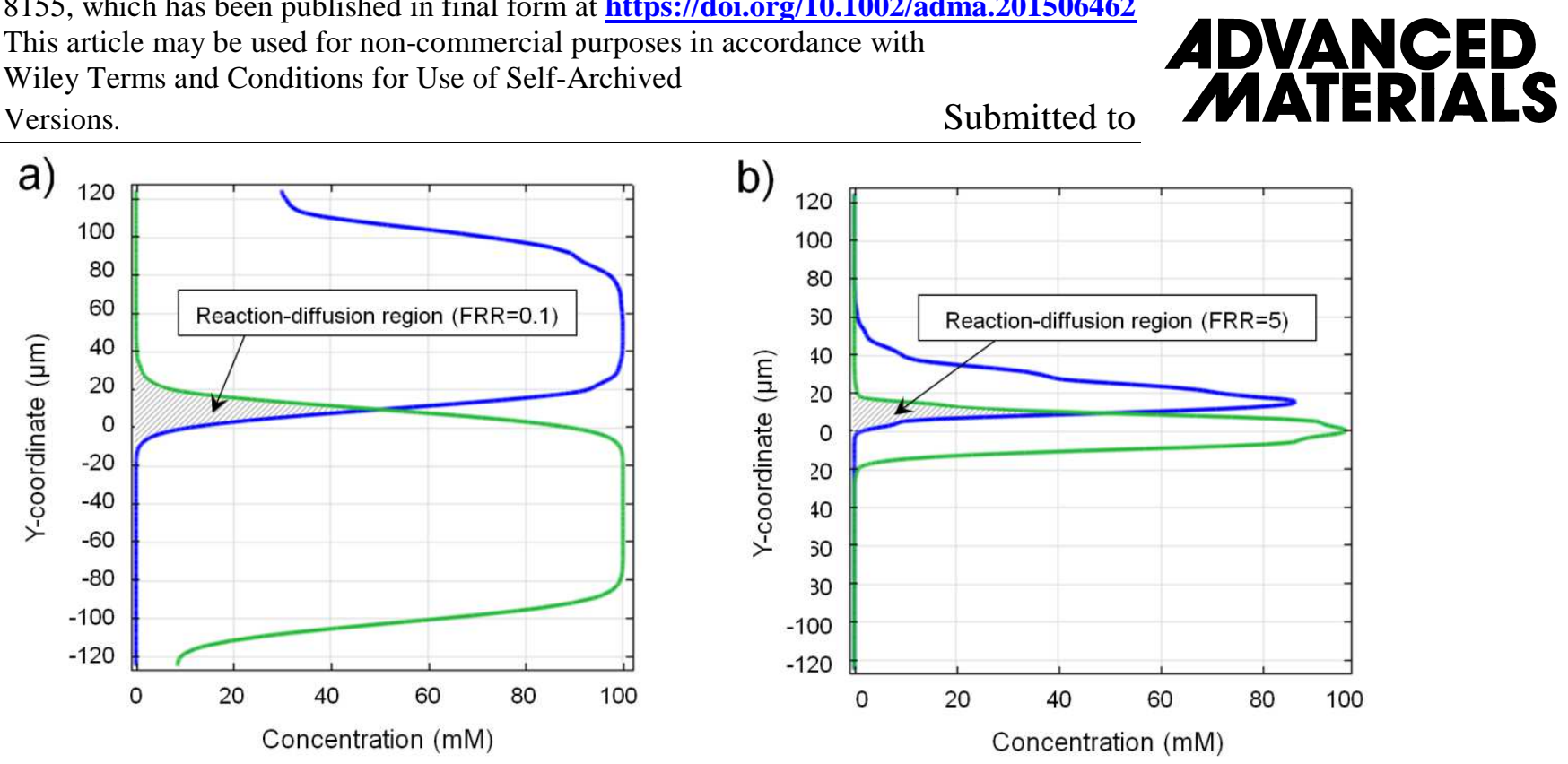

Figure SI.1 | Concentration profiles of $\mathrm{Cu}\left(\mathrm{NO}_{3}\right)_{2} \cdot 6 \mathrm{H}_{2} \mathrm{O}$ (blue) and 4,4'-bpy (green) at the microfluidic reactor exit at $\mathrm{FRR}=0.1(\mathrm{TFR}=1,200 \mu \mathrm{L} / \mathrm{min})(\mathbf{a})$ and $\mathrm{FRR}=5(\mathrm{TFR}=220 \mu \mathrm{L} / \mathrm{min})$ (b) calculated using constant values of the reagents flow rates $\left(\mathrm{Q}_{2}\right.$ and $\left.\mathrm{Q}_{3}=100 \mu \mathrm{L} / \mathrm{min}\right)$. In the reaction-diffusion region both reagents are present. 
This is the peer reviewed version of the following article: Marta Rubio-Martinez ... [et al.], Freezing the nonclassical crystal growth of a coordination polymer using controlled dynamic gradients. Adv. Mater., 28: 81508155, which has been published in final form at https://doi.org/10.1002/adma.201506462

This article may be used for non-commercial purposes in accordance with

Wiley Terms and Conditions for Use of Self-Archived

Versions.

Submitted to

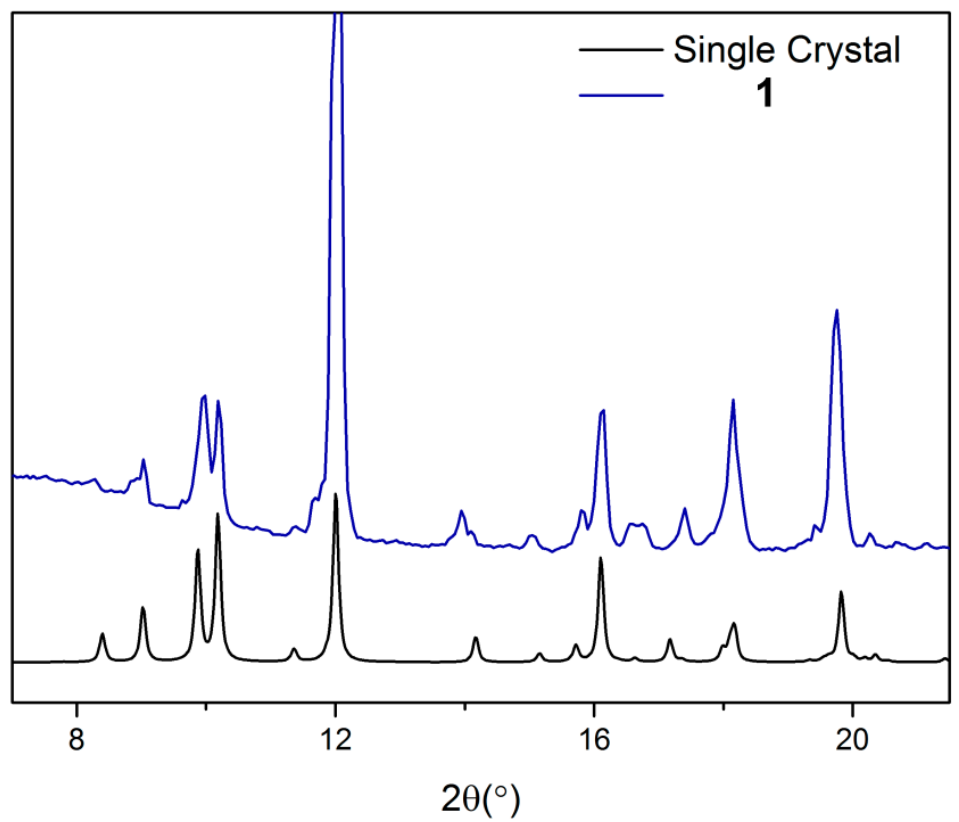

Figure SI.2 | Comparison of the experimental XRPD pattern (blue) of the microcrystals of 1 obtained by simple mixture of $\mathrm{Cu}\left(\mathrm{NO}_{3}\right)_{2} \cdot 6 \mathrm{H}_{2} \mathrm{O}$ and 4,4'-bpy with the XRPD pattern simulated from its single-crystal structure (black). 
This is the peer reviewed version of the following article: Marta Rubio-Martinez ... [et al.], Freezing the nonclassical crystal growth of a coordination polymer using controlled dynamic gradients. Adv. Mater., 28: 81508155, which has been published in final form at https://doi.org/10.1002/adma.201506462

This article may be used for non-commercial purposes in accordance with

Wiley Terms and Conditions for Use of Self-Archived

Versions.

Submitted to

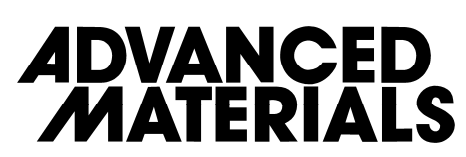

\section{FRR = 5}

$\mathrm{TFR}=$

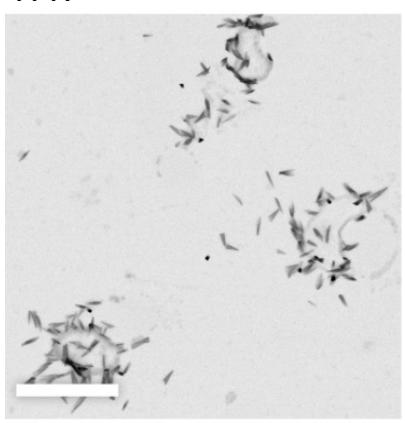

$F R R=2$

TFR =
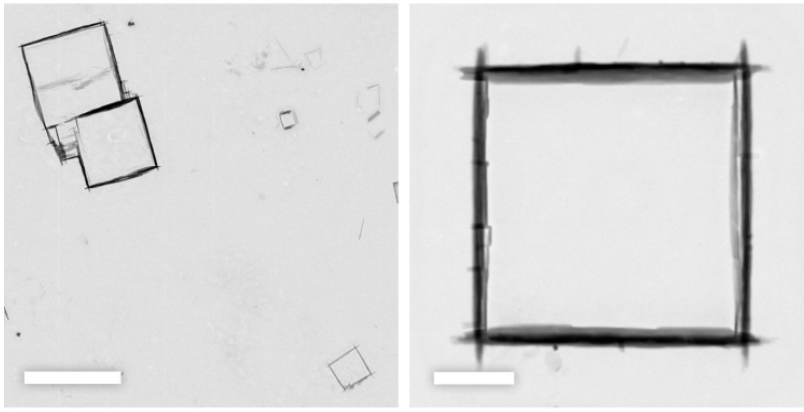

$F R=0.1$

TFR =

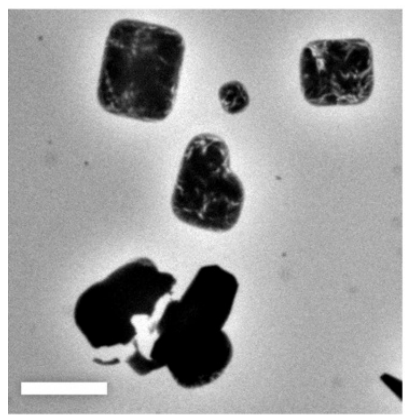

$\mathrm{TFR}=600$

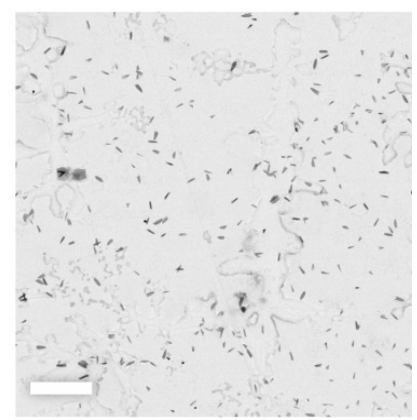

TFR $=$

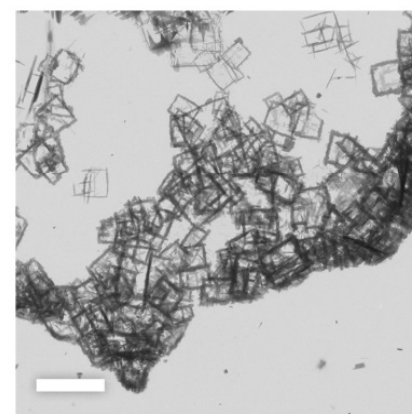

TFR =

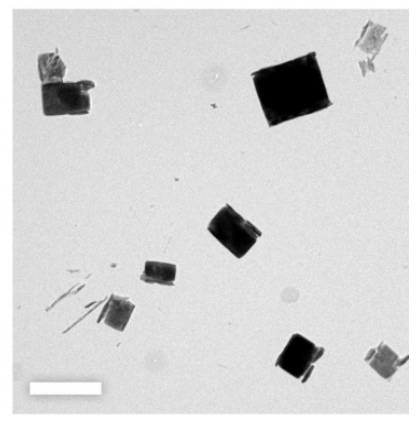

Figure SI.3 | TEM images of crystals of $\mathbf{1}$ fabricated in the microfluidic device at FRR = 5, 2 and 0.1 , respectively, from top to down. Note that different TFR combinations have been used to work under the same FRR. Also note that identical crystalline phases -from needles to hollow frames to plate-like crystals (top to down)- have been synthesized for the same FRR values, independently of the flow rates used. The scale bars are $2 \mu \mathrm{m}$ (top row), $5 \mu \mathrm{m}$ (left and right 
This is the peer reviewed version of the following article: Marta Rubio-Martinez ... [et al.], Freezing the nonclassical crystal growth of a coordination polymer using controlled dynamic gradients. Adv. Mater., 28: 81508155, which has been published in final form at https://doi.org/10.1002/adma.201506462

This article may be used for non-commercial purposes in accordance with

Wiley Terms and Conditions for Use of Self-Archived

Versions.

Submitted to

ADVANCED

middle row), $2 \mu \mathrm{m}$ (centred middle row), $10 \mu \mathrm{m}$ (left and right bottom row) and $4 \mu \mathrm{m}$ (centred

bottom row). 
This is the peer reviewed version of the following article: Marta Rubio-Martinez ... [et al.], Freezing the nonclassical crystal growth of a coordination polymer using controlled dynamic gradients. Adv. Mater., 28: 81508155, which has been published in final form at https://doi.org/10.1002/adma.201506462

This article may be used for non-commercial purposes in accordance with

Wiley Terms and Conditions for Use of Self-Archived Versions.

a)

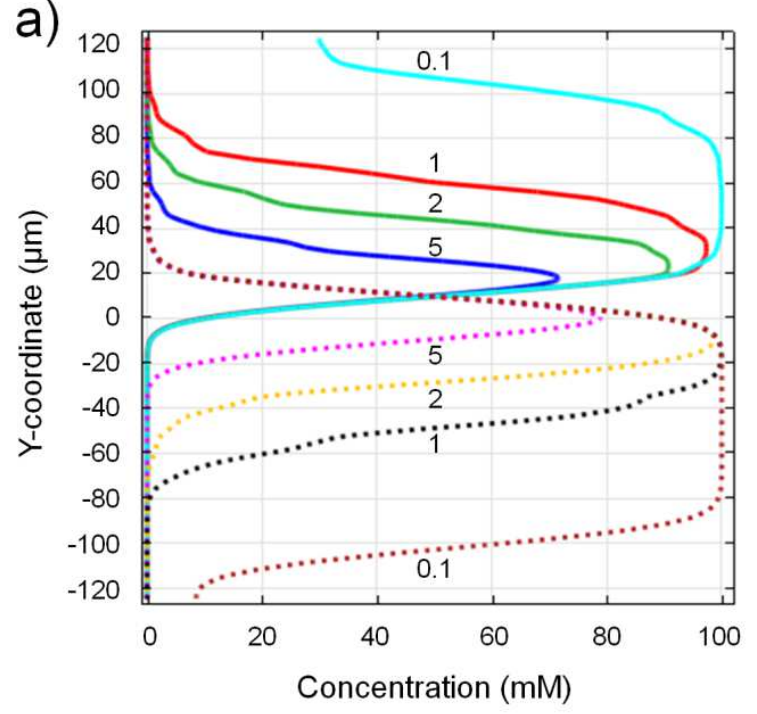

Submitted to

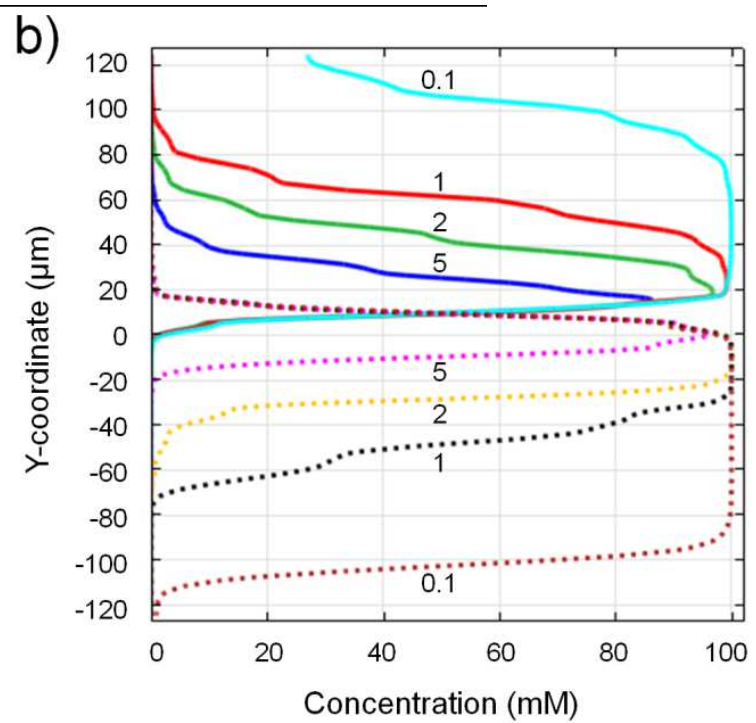

Figure SI.4 | Concentration profiles of reagents at the microfluidic reactor exit, for increasing values of flow rate ratio ( $F R R=0.1,1,2$ and 5) and two values of total flow rate i.e. $\mathrm{TFR}=220 \mu \mathrm{L} / \mathrm{min}$ (a) and 1,200 $\mu \mathrm{L} / \mathrm{min})(\mathbf{b})$. Solid and dashed lines correspond to concentration profiles of $\mathrm{Cu}\left(\mathrm{NO}_{3}\right)_{2} \cdot 6 \mathrm{H}_{2} \mathrm{O}$ and $4,4^{\prime}$-bpy, respectively. 
This is the peer reviewed version of the following article: Marta Rubio-Martinez ... [et al.], Freezing the nonclassical crystal growth of a coordination polymer using controlled dynamic gradients. Adv. Mater., 28: 81508155, which has been published in final form at https://doi.org/10.1002/adma.201506462

This article may be used for non-commercial purposes in accordance with

Wiley Terms and Conditions for Use of Self-Archived

Versions.

Submitted to

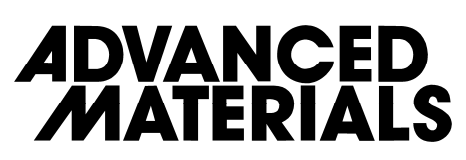

a)

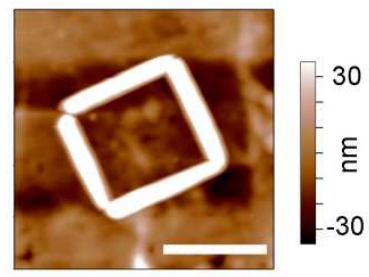

d)

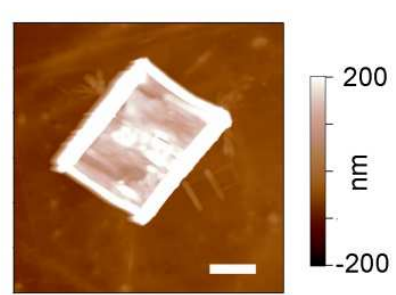

b)

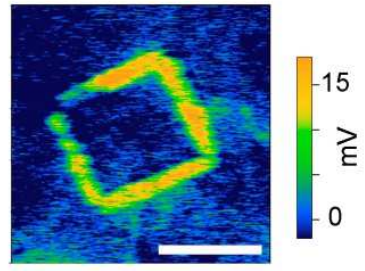

e)

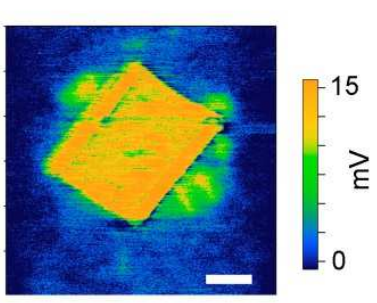

c)

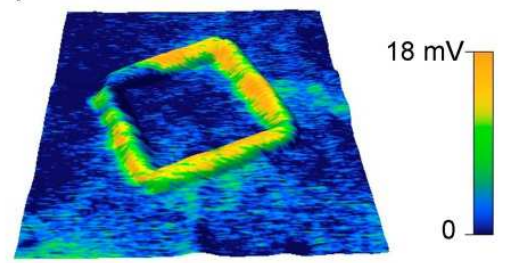

f)

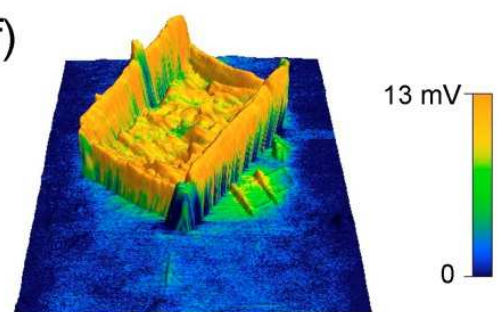

Figure SI.5 | AFM topography images of a hollow frame (a) and a partially filled frame (d). Kelvin Probe Microscopy images (KPFM); b,e, 2D and c,f, 3D AFM topography images with KPFM signal as the colour scale. The surface potential function difference measured by KPFM confirms the absence of $\mathrm{CP}$ material inside of the frame. 
This is the peer reviewed version of the following article: Marta Rubio-Martinez ... [et al.], Freezing the nonclassical crystal growth of a coordination polymer using controlled dynamic gradients. Adv. Mater., 28: 81508155, which has been published in final form at https://doi.org/10.1002/adma.201506462

This article may be used for non-commercial purposes in accordance with

Wiley Terms and Conditions for Use of Self-Archived

Versions.

Submitted to

\section{ADVANCED MATERIALS}
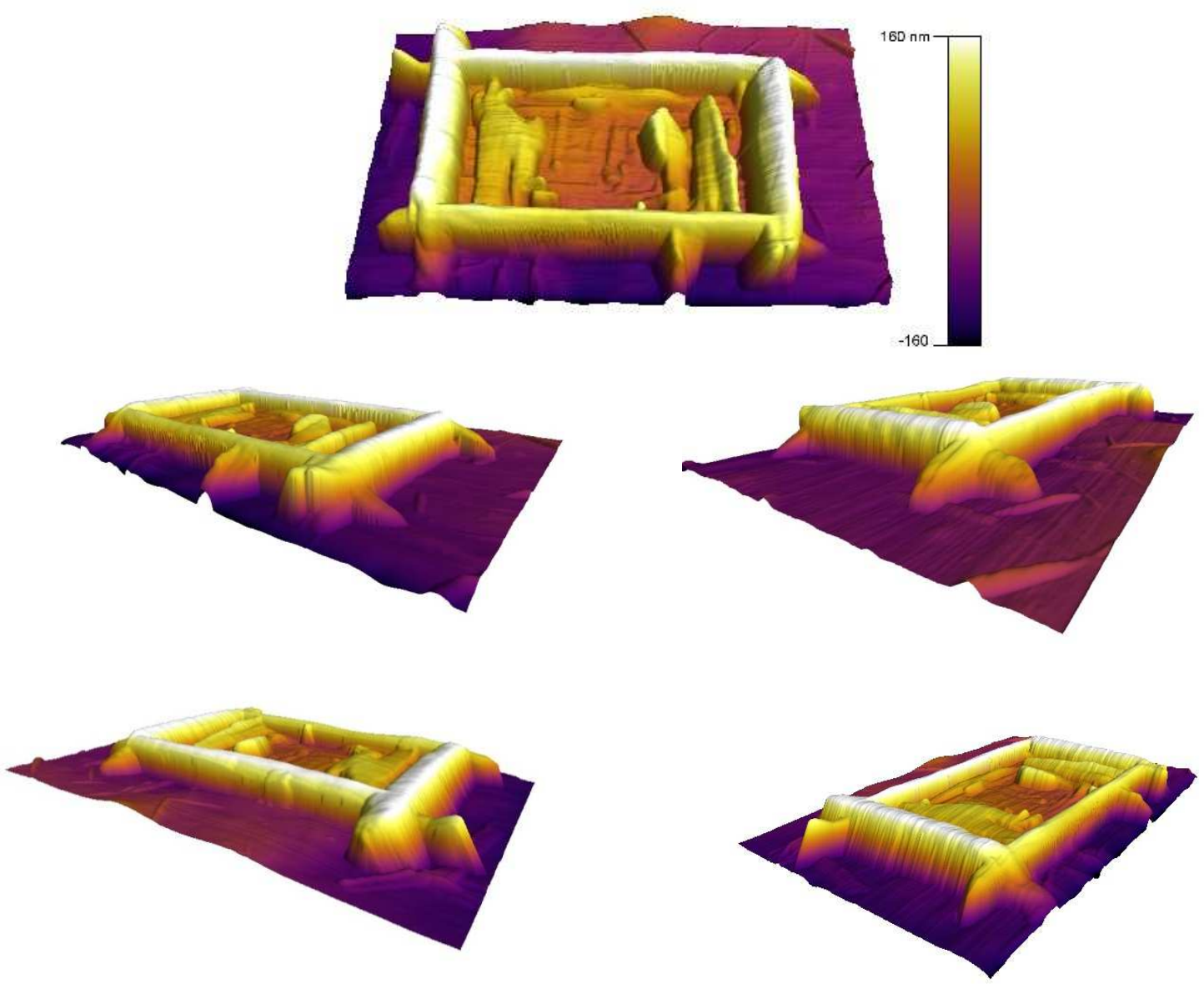

Figure SI.6 | 3D AFM topography images of a single frame, showing different perspectives for the vertices. Note that the vertices are interpenetrated. 
This is the peer reviewed version of the following article: Marta Rubio-Martinez ... [et al.], Freezing the nonclassical crystal growth of a coordination polymer using controlled dynamic gradients. Adv. Mater., 28: 81508155, which has been published in final form at https://doi.org/10.1002/adma.201506462

This article may be used for non-commercial purposes in accordance with

Wiley Terms and Conditions for Use of Self-Archived

Versions.

Submitted to
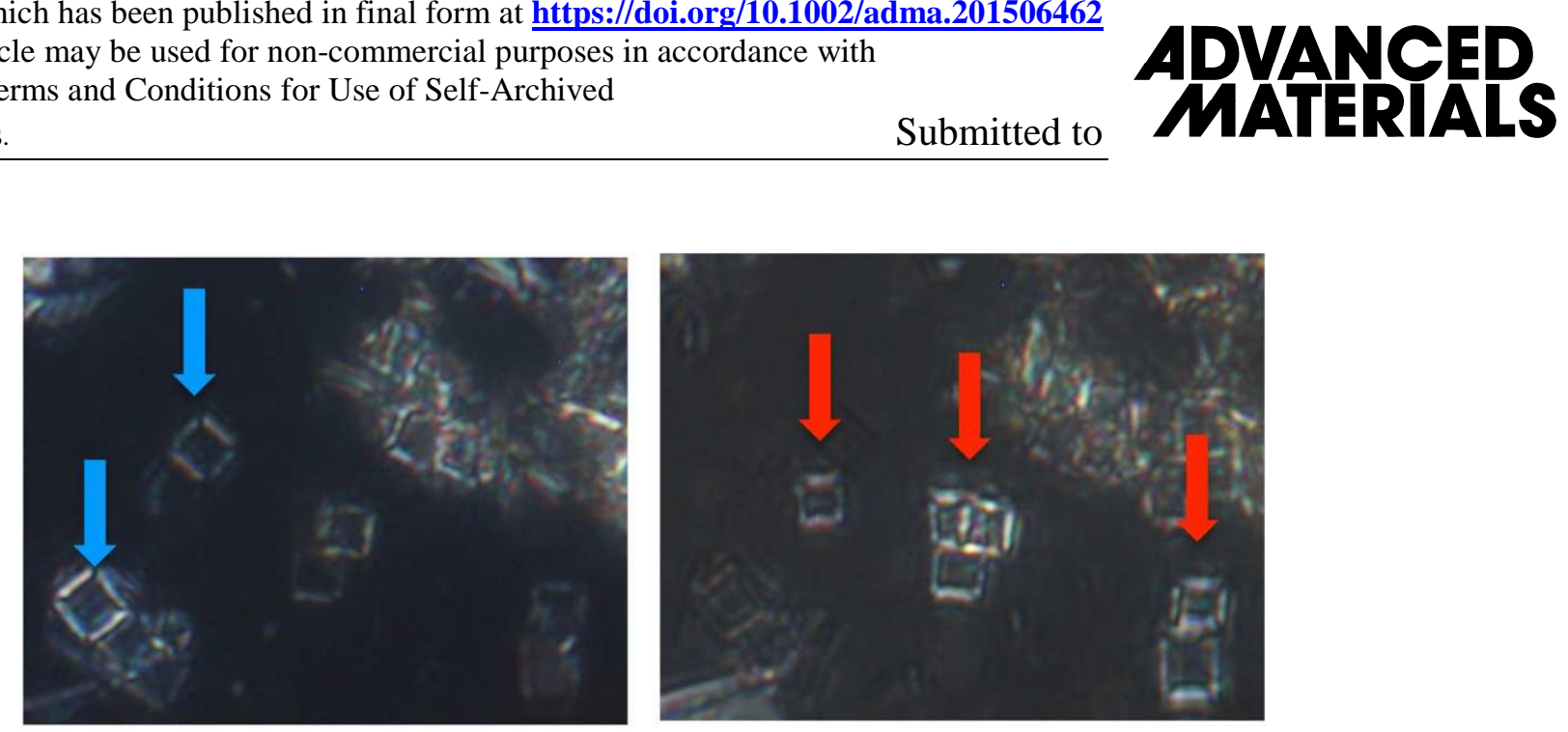

$5 \mu \mathrm{m}$

Figure SI.7 | POM images showing the optical axes of two frames that indicate that the four sides have perfect orientational order.

\section{References}

[1] Z. Otwinowski, W. Minor, in Method Enzym., Elsevier, 1997, pp. 307-326.

[2] G. M. Sheldrick, Acta Crystallogr. A 2008, 64, 112.

[3] K. C. Pratt, W. A. Wakeham, Proc. R. Soc. Lond. Math. Phys. Eng. Sci. 1974, 336, 393. 